Pacific

Journal of

Mathematics

ANGULAR DERIVATIVES OF BOUNDED UNIVALENT FUNCTIONS AND EXTREMAL PARTITIONS OF THE UNIT DISK

Christian Pommerenke and Alexander Vasil'ev 


\title{
ANGULAR DERIVATIVES OF BOUNDED UNIVALENT FUNCTIONS AND EXTREMAL PARTITIONS OF THE UNIT DISK
}

\author{
Christian Pommerenke and Alexander Vasil'ev
}

\begin{abstract}
We study bounded univalent functions $f(z)$ that map the unit disk into itself such that $f(0)=0$ and the angular limits $f\left(\zeta_{k}\right)$ with the angular derivatives $f^{\prime}\left(\zeta_{k}\right)$ exist at fixed points $\zeta_{k}$ of the unit circle, $k=1, \ldots, n$. We use a general inequality of Schiffer-Tammi type obtained earlier by the authors and discuss the cases of the equality sign. Sharp estimates of functionals are obtained in classes of such functions. An explicit form of extremal functions is deduced. Since one of the methods of solution is based on the extremal partition of the unit disk, we are also concerned with some geometric problems. In particular, we study the problem of the maximum of the sum of the reduced moduli of digons and circular domains. As a corollary we derive sharp estimates of functionals dependent on $\left(\left|f^{\prime}(0)\right|,\left|f^{\prime}\left(\zeta_{1}\right) f^{\prime}\left(\zeta_{2}\right)\right|\right)$.
\end{abstract}

\section{Introduction and preliminaries.}

We denote by $\mathbb{D}$ the unit disk $\{z:|z|<1\}$ and set $\mathbb{T}:=\partial \mathbb{D}$. An important notion in the problems of the boundary behavior of conformal maps (see [10]) is the Stolz angle at a point $\zeta \in \mathbb{T}$ that is of the form

$$
\begin{aligned}
\Delta_{\zeta}=\{z \in \mathbb{D}:|\arg (1-\bar{\zeta} z)|<\theta,|z-\zeta|<\eta\}, \\
\text { with } \theta \in\left(0, \frac{\pi}{2}\right), \eta \in(0,2 \cos \theta) .
\end{aligned}
$$

We say that $f$ has the angular limit $a \in \overline{\mathbb{C}}$ at $\zeta \in \mathbb{T}$ if $f(\zeta) \rightarrow a$ as $z \in \Delta_{\zeta}$, $z \rightarrow \zeta$ for any Stolz angle $\Delta_{\zeta}$ at $\zeta$. We denote this angular limit by $f(\zeta)$. If the limit $f(z) \rightarrow a$ exists for all $z \in \mathbb{D}, z \rightarrow \zeta$, then $f$ becomes continuous at $\zeta$ as a function in $\mathbb{D} \cup\{\zeta\}$. As one can see in $[10]$ univalent functions in $\mathbb{D}$ have the angular limits at almost all points in $\mathbb{T}$ but are continuous there only in some restricted cases. Let us consider functions $f$ that map the disk $\mathbb{D}$ into itself. Then the angular limit $f(\zeta)$ exists for almost all $\zeta \in \mathbb{T}$. Moreover, the exceptional set in $\mathbb{T}$ has zero capacity. 
We say that $f$ has the angular derivative $f^{\prime}(\zeta)$ at $\zeta \in \mathbb{T}$ if the finite angular limit $f(\zeta)$ exists and if

$$
\lim _{z \rightarrow \zeta, z \in \Delta_{\zeta}} \frac{f(z)-f(\zeta)}{z-\zeta}=f^{\prime}(\zeta)
$$

The angular derivative $f^{\prime}(\zeta)$ exists if and only if the analytic function $f^{\prime}(z)$ has the angular limit $f^{\prime}(\zeta)$ (see [10, Proposition 4.7]). Generally very little can be said about the existence of the angular derivative. However, for selfmaps of $\mathbb{D}$ the Julia-Wolff lemma (see [10, Proposition 4.13]) implies that the angular derivative $f^{\prime}(\zeta)$ exists for all points $\zeta$ where the angular limit $f(\zeta)$ exists and $|f(\zeta)|=1$ even without assumption of univalence. Furthermore, for the case of univalent functions the McMillan Twist Theorem [8] says that the angular derivative is finite at almost all such points. Thus, the points $\zeta$ such that $|f(\zeta)|=1$ are of particular interest.

In $[\mathbf{1 1}]$ we studied some extremal problems for univalent functions $f$ : $\mathbb{D} \rightarrow \mathbb{D}$ such that $f(0)=0$ and the angular limits (whose absolute value is equal to 1) exist as well as the finite angular derivatives at certain points of $\mathbb{T}$. Several inequalities have been derived to obtain sharp estimates of functionals dependent on the angular derivatives. We have proved that the functions are bilipschitz in the set of $\mathbb{T}$ where the angular limits exist as well as the finite angular derivatives. In particular, we have considered the noncompact class of functions $f(z): \mathbb{D} \rightarrow \mathbb{D}$ such that $f(0)=0, f(1)=1$ with the angular derivative $f^{\prime}(1)$ fixed. We have found the sharp lower estimate $\left|f^{\prime}(1)\right| \geq 1 / \sqrt{\left|f^{\prime}(0)\right|}$, the lower estimates of $|f(z)|$ dependent on the value of $\left|f^{\prime}(0)\right|$ fixed. In [17] A. Vasil'ev has used the extremal partition of $\mathbb{D}$ by digons to deduce the sharp lower boundary curve for the range of the system of functionals $\left(|f(r)|,\left|f^{\prime}(r)\right|\right), 0<r<1$.

In $[\mathbf{1 1}$, Theorem 2.1] we have derived two positive semi-definite quadratic forms from the Schiffer-Tammi analog [12] for the Grunsky inequality (compare [9, Corollary 4.3]). $\S 2$ is devoted to the explicit form of the extremal functions for these forms to vanish. As a corollary we obtain the sharp estimate of a functional dependent on $\left|f^{\prime}\left(\zeta_{1}\right)\right|$ and $\left|f^{\prime}\left(\zeta_{2}\right)\right|$. Namely, we will derive the inequality

$$
\left|\frac{f\left(\zeta_{1}\right)-f\left(\zeta_{2}\right)}{\zeta_{1}-\zeta_{2}}\right|^{2 t_{1} t_{2}} \geq \frac{1}{\sqrt{\left|f^{\prime}(0)\right|\left|f^{\prime}\left(\zeta_{1}\right)\right| t_{1}^{2}\left|f^{\prime}\left(\zeta_{2}\right)\right|^{t_{2}^{2}}}},
$$

which is sharp for all $\zeta_{1}, \zeta_{2} \in \mathbb{T}$ and $t_{1}, t_{2} \geq 0, t_{1}+t_{2}=1$. In particular, for $t_{1}=t_{2}=1 / 2$ we have the inequality [11, Corollary 3.3]. The extremal functions will map $\mathbb{D}$ onto $\mathbb{D}$ with one or two analytic slits.

An important part of the theory of conformal maps is conformal invariants and various notions connected with them. One of the topical conformal invariants is the modulus of a doubly connected hyperbolic domain in the Riemann sphere $\overline{\mathbb{C}}$ or a Riemann surface. This modulus remains the same 
under a conformal map. Another quantity which is very much used in the theory of distortion is the reduced modulus $m(D, a)$ of a simply connected hyperbolic domain $D$ with respect to a fixed point $a \in D$ which we call a circular domain. Let the symbol $R(D, a)$ stand for the conformal (interior) radius of a domain $D \subset \overline{\mathbb{C}}$ with respect to a point $a$ with $a \neq \infty$, or $R(D, \infty)=e^{\gamma}$ where $\gamma$ is the Robin constant otherwise. Then $m(D, a)=$ $\frac{1}{2 \pi} \log R(D, a)$ or $m(D, \infty)=-\frac{1}{2 \pi} \log \operatorname{cap}(\partial D)$ (see e.g., $\left.[\mathbf{3}, \mathbf{5}, \mathbf{6}]\right)$. If $f$ is a conformal map from $D$ into $\overline{\mathbb{C}}, f(a) \neq \infty$, then the reduced modulus changes according to the rule $m(D, a)+\frac{1}{2 \pi} \log \left|f^{\prime}(a)\right|=m(f(D), f(a))$. Or, if $D$ is a simply connected hyperbolic domain, $a \in D,|a|<\infty$ and $f(z)=a_{-1} /(z-a)+a_{0}+a_{1}(z-a)+\ldots$ is a conformal map from $D$, then $m(f(D), \infty)=m(D, a)-\frac{1}{2 \pi} \log \left|a_{-1}\right|$.

We are concerned also with another quantity that has appeared rather recently in $[\mathbf{2}, \mathbf{7}, \mathbf{1 4}]$ and now is used actively for extremal problems for conformal maps (see $[\mathbf{2}, \mathbf{7}, \mathbf{1 6}, \mathbf{1 7}]$ ) which is called the reduced modulus of digon. Let us mention here that a discussion about the term digon-bigonbiangle that has appeared in $[\mathbf{1 1}$, p. 89] does not belong to the authors and has been added to the final version of the paper by the editor of the journal. Now we define the reduced moduli of digons. For details we refer to the papers by E.G. Emel'yanov [2], G.V. Kuz'mina [7], some recent results can be read in $[\mathbf{1 4}]$.

Let $D$ be a hyperbolic simply connected domain in $\mathbb{C}$ with two finite fixed boundary points $a, b$ (maybe with the same support) on its piecewise smooth boundary. It is called a digon. We set the region $S(a, \varepsilon)$ that is a connected component of $D \cap\{|z-a|<\varepsilon\}$ with the point $a$ on its border. Denote by $D_{\varepsilon}$ the domain $D \backslash\left\{S\left(a, \varepsilon_{1}\right) \cup S\left(b, \varepsilon_{2}\right)\right\}$ for sufficiently small $\varepsilon_{1,2}$. Denote by $M\left(D_{\varepsilon}\right)$ the modulus of the family of arcs in $D_{\varepsilon}$ joining the boundary arcs of $S\left(a, \varepsilon_{1}\right)$ and $S\left(b, \varepsilon_{2}\right)$ that lie in the circumferences $|z-a|=\varepsilon_{1}$ and $|z-b|=\varepsilon_{2}$ (we choose a single arc in each circle so that both arcs can be connected in $\left.D_{\varepsilon}\right)$. If the limit

$$
m(D, a, b)=\lim _{\varepsilon_{1,2} \rightarrow 0}\left(\frac{1}{M\left(D_{\varepsilon}\right)}+\frac{1}{\varphi_{a}} \log \varepsilon_{1}+\frac{1}{\varphi_{b}} \log \varepsilon_{2}\right)
$$

exists, where $\varphi_{a}=\sup \Delta_{a}$ and $\varphi_{b}=\sup \Delta_{b}$ are the inner angles and $\Delta_{a, b}$ is the Stolz angle inscribed in $D$ at $a$ or $b$ respectively, then it is called the reduced modulus of the digon $D$. Various conditions guarantee the existence of this modulus, however, even in the case of piecewise analytic boundary there are examples [14] which show that it is not always the case. The existence of the limit (1.2) is a local characteristic of the domain $D$ (see [14, Theorem 1.2]). If the domain $D$ is conformal (see the definition in $[\mathbf{1 0}$, p. 80]) at the points $a$ and $b$, then $[\mathbf{1 4}$, Theorem 1.3] this condition is sufficient for the limit (1.2) to exist. 
More generally, suppose that there exists a conformal map $f(z)$ of the domain $S\left(a, \varepsilon_{1}\right) \subset D$ onto a circular sector so that there exists the angular limit $f(a)$ which is the vertex of this sector and with the angle $\varphi_{a}$. If the function $f$ has the angular finite nonzero derivative $f^{\prime}(a)$ we say that the domain $D$ is also conformal at the point a (compare [10, p. 80]). If the digon $D$ is conformal at the points $a, b$, then the limit (1.2) exists [14].

Suppose that there exists a conformal map $f(z)$ of the digon $D$ (which is conformal at $a, b)$ onto a digon $D^{\prime}$ so that there exist the angular limits $f(a), f(b)$ with the inner angles $\psi_{a}$ and $\psi_{b}$ at the vertices $f(a)$ and $f(b)$ which we also understand as the supremum over all Stolz angles inscribed in $D^{\prime}$ with the vertices at $f(a)$ or $f(b)$ respectively. If the function $f$ has the angular finite nonzero derivatives $f^{\prime}(a)$ and $f^{\prime}(b)$ then $\varphi_{a}=\psi_{a}, \varphi_{b}=\psi_{b}$, and the reduced modulus (1.2) exists and changes according to the rule

$$
m(f(D), f(a), f(b))=m(D, a, b)+\frac{1}{\psi_{a}} \log \left|f^{\prime}(a)\right|+\frac{1}{\psi_{b}} \log \left|f^{\prime}(b)\right| .
$$

If we suppose, moreover, that $f$ has the expansion

$$
f(z)=w_{1}+(z-a)^{\psi_{a} / \varphi_{a}}\left(c_{1}+c_{2}(z-a)+\ldots\right)
$$

in a neighborhood of the point $a$, and the expansion

$$
f(z)=w_{2}+(z-b)^{\psi_{b} / \varphi_{b}}\left(d_{1}+d_{2}(z-a)+\ldots\right)
$$

in a neighborhood of the point $b$, then the reduced modulus of $D$ changes according to the rule

$$
m(f(D), f(a), f(b))=m(D, a, b)+\frac{1}{\psi_{a}} \log \left|c_{1}\right|+\frac{1}{\psi_{b}} \log \left|d_{1}\right| .
$$

Obviously, one can extend this definition to the case of vertices with the infinite support.

We also consider a quantity which is called the reduced modulus of a triangle. It closely connected with the reduced modulus of a digon. For details we refer to the paper by A.Yu. Solynin [14].

Let $D$ be a hyperbolic simply connected domain in $\mathbb{C}$ with three finite fixed boundary points $z_{1}, z_{2}$, and $a$ on its piecewise smooth boundary. Denote by $D_{\varepsilon}$ the domain $D \backslash S\left(a, \varepsilon_{1}\right)$ for a sufficiently small $\varepsilon$. Denote by $M\left(D_{\varepsilon}\right)$ the modulus of the family of arcs in $D_{\varepsilon}$ joining the boundary arc of $S(a, \varepsilon)$ that lies in the circumference $|z-a|=\varepsilon$ with the leg of the triangle $D$ which is opposite to $a$. If the limit

$$
m_{\Delta}(D, a)=\lim _{\varepsilon \rightarrow 0}\left(\frac{1}{M\left(D_{\varepsilon}\right)}+\frac{1}{\varphi_{a}} \log \varepsilon\right)
$$

exists, where $\varphi_{a}$ is the inner angle defined as before, then it is called the reduced modulus of the triangle $D$. The conditions for the reduced modulus to exist are similar to those for the reduced modulus of digons. It turns out that the reduced modulus exists if $D$ is conformal at $a$. If there exists 
a conformal map $f(z)$ from the triangle $D$ onto a triangle $D^{\prime}$ so that there exists the angular limit $f(a)$ with the inner angle $\psi_{a}$ at the vertex $f(a)$, if the function $f$ has the angular finite nonzero derivative $f^{\prime}(a)$, then $\varphi_{a}=\psi_{a}$ and the reduced modulus of $D$ exists and changes $[\mathbf{1 4}]$ according to the rule

$$
m_{\Delta}(f(D), f(a))=m_{\Delta}(D, a)+\frac{1}{\psi_{a}} \log \left|f^{\prime}(a)\right| .
$$

If we suppose, moreover, that $f$ has the expansion

$$
f(z)=w_{1}+(z-a)^{\psi_{a} / \varphi_{a}}\left(c_{1}+c_{2}(z-a)+\ldots\right)
$$

in a neighborhood of the point $a$, then the reduced modulus of $D$ changes according to the rule

$$
m_{\Delta}(f(D), f(a))=m_{\Delta}(D, a)+\frac{1}{\psi_{a}} \log \left|c_{1}\right| .
$$

In $\S 3$ we consider two cases of extremal partitions of $\mathbb{D}$ by digons and circular domains, and calculate the extremal value of the weighted sum of their reduced moduli.

In $\S 4$ we apply the results of $\S 3$ to derive some sharp estimates for functionals in the class of bounded univalent functions with the angular derivatives fixed. Also we give there another proof of (1.1) to examplify the method.

In many cases, the extremal function is the classical conformal Pick map

$$
p_{\alpha}(z)=\frac{4 \alpha z}{\left(1-z+\sqrt{(1-z)^{2}+4 \alpha z}\right)^{2}}=\alpha z+\ldots
$$

of $\mathbb{D}$ onto $\mathbb{D} \backslash\left[-1,-\alpha /(1+\sqrt{1-\alpha})^{2}\right]$. It satisfies the identities

$$
\frac{p_{\alpha}(z)}{\left(1-p_{\alpha}(z)\right)^{2}}=\frac{\alpha z}{(1-z)^{2}}, \quad \frac{p_{\alpha}(z)}{\left(1+p_{\alpha}(z)\right)^{2}}=\frac{\alpha z}{(1-z)^{2}+4 \alpha z} \text {. }
$$

We denote rotations of the Pick function by $g_{\alpha}^{\varkappa}(z) \equiv e^{i \varkappa} p_{\alpha}\left(e^{-i \varkappa} z\right)$ specifying every time the angle $\varkappa$.

\section{The equality in the Schiffer-Tammi inequality.}

Let the function $f(z)=\alpha z+\ldots(0<\alpha \leq 1)$ be univalent in $\mathbb{D}$ and let $f(\mathbb{D}) \subset \mathbb{D}$. We define $a_{j k}=a_{k j}(j, k=0,1, \ldots)$ and $a_{j k}^{*}=\overline{a_{k j}^{*}}(j, k=$ $1,2, \ldots)$ by

$$
\begin{aligned}
\log \frac{f(z)-f(\zeta)}{z-\zeta} & =\sum_{j=0}^{\infty} \sum_{k=0}^{\infty} a_{j k} z^{j} \zeta^{k}, \quad(z, \zeta \in \mathbb{D}), \\
-\log [1-f(z) \overline{f(\zeta)}] & =\sum_{j=1}^{\infty} \sum_{k=1}^{\infty} a_{j k}^{*} z^{j} \bar{\zeta}^{k}, \quad(z, \zeta \in \mathbb{D}) .
\end{aligned}
$$


We mention that here and further on the removable singularity has been removed. M. Schiffer and O. Tammi [12] have shown that

$$
\operatorname{Re}\left[\sum_{j=0}^{\infty} \sum_{k=0}^{\infty} a_{j k} \lambda_{j} \lambda_{k}\right]+\sum_{j=1}^{\infty} \sum_{k=1}^{\infty} a_{j k}^{*} \lambda_{j} \bar{\lambda}_{k} \leq \sum_{k=1}^{\infty} \frac{\left|\lambda_{k}\right|^{2}}{k}
$$

for $\lambda_{0} \in \mathbb{R}, \lambda_{k} \in \mathbb{C}(k=1,2, \ldots)$; the case $\lambda_{0}=0$ is due to Z. Nehari (see also $[\mathbf{9}$, Theorem 4.2]).

In [11] we have derived two positive semi-definite quadratic forms from the Schiffer-Tammi inequality (2.3); compare [9, Corollary 4.3]. For the completeness we also give here the proof.

Theorem 2.1 ([11, Theorem 2.1]). Let $f(z)=\alpha z+\ldots$ be univalent in $\mathbb{D}$ and $f(\mathbb{D}) \subset \mathbb{D}$. If $z_{\nu} \in \mathbb{D}$ and $w_{\nu}=f\left(z_{\nu}\right)$ for $\nu=1, \ldots, n$ and if $x_{\nu} \in \mathbb{R}$, $(\nu=0, \ldots, n)$, then

$$
\begin{aligned}
x_{0}^{2} \log \frac{1}{\alpha}+2 & \sum_{\nu=1}^{n} x_{0} x_{\nu} \arg \frac{w_{\nu}}{z_{\nu}} \\
& +\sum_{\mu=1}^{n} \sum_{\nu=1}^{n} x_{\mu} x_{\nu} \log \left|\frac{\alpha z_{\mu} z_{\nu}}{w_{\mu} w_{\nu}} \cdot \frac{w_{\mu}-w_{\nu}}{z_{\mu}-z_{\nu}} \cdot \frac{1-w_{\mu} \bar{w}_{\nu}}{1-z_{\mu} \bar{z}_{\nu}}\right| \geq 0,
\end{aligned}
$$

and

$$
\begin{aligned}
x_{0}^{2} \log \frac{1}{\alpha}+2 \sum_{\nu=1}^{n} x_{0} x_{\nu} \log \left|\frac{w_{\nu}}{z_{\nu}}\right| & \\
& +\sum_{\mu=1}^{n} \sum_{\nu=1}^{n} x_{\mu} x_{\nu} \log \left|\frac{z_{\mu}-z_{\nu}}{w_{\mu}-w_{\nu}} \cdot \frac{1-w_{\mu} \bar{w}_{\nu}}{1-z_{\mu} \bar{z}_{\nu}}\right| \geq 0,
\end{aligned}
$$

where by $\left(w_{\mu}-w_{\nu}\right) /\left(z_{\mu}-z_{\nu}\right)$ we understand the derivative $f^{\prime}\left(z_{\mu}\right)$ in the case $\mu=\nu$.

Proof. (a) First we set

$$
\lambda_{0}=-x_{0}, \lambda_{k}=i \sum_{\nu=1}^{n} x_{\nu} z_{\nu}^{k}, \quad(k=1,2, \ldots)
$$

and obtain from the definitions (2.1) and (2.2) that

$$
a_{00}=\log \alpha, \sum_{k=1}^{\infty} a_{k 0} \lambda_{k}=i \sum_{\nu=1}^{n} x_{\nu} \log \frac{w_{\nu}}{\alpha z_{\nu}}
$$




$$
\begin{aligned}
\sum_{j=1}^{\infty} \sum_{k=1}^{\infty} a_{j k} \lambda_{j} \lambda_{k} & =-\sum_{\mu=1}^{n} \sum_{\nu=1}^{n} x_{\mu} x_{\nu} \log \left(\frac{\alpha z_{\mu} z_{\nu}}{w_{\mu} w_{\nu}} \cdot \frac{w_{\mu}-w_{\nu}}{z_{\mu}-z_{\nu}}\right), \\
\sum_{j=1}^{\infty} \sum_{k=1}^{\infty} a_{j k}^{*} \lambda_{j} \bar{\lambda}_{k} & =-\sum_{\mu=1}^{n} \sum_{\nu=1}^{n} x_{\mu} x_{\nu} \log \left(1-w_{\mu} \bar{w}_{\nu}\right), \\
\sum_{k=1}^{\infty} \frac{1}{k}\left|\lambda_{k}\right|^{2} & =-\sum_{\mu=1}^{n} \sum_{\nu=1}^{n} x_{\mu} x_{\nu} \log \left(1-z_{\mu} \bar{z}_{\nu}\right) .
\end{aligned}
$$

Hence (2.4) follows from the Schiffer-Tammi inequality (2.3).

(b) Now we set

$$
\lambda_{0}=-x_{0}+\sum_{\nu=1}^{n} x_{\nu}, \quad \lambda_{k}=\sum_{\nu=1}^{n} x_{\nu} z_{\nu}^{k}, \quad(k=1,2, \ldots) .
$$

We obtain from (2.1) and (2.3) that

$\sum_{j=1}^{\infty} \sum_{k=1}^{\infty} a_{j k} \lambda_{j} \lambda_{k}=x_{0}^{2} \log \alpha-2 x_{0} \sum_{\nu=1}^{n} x_{\nu} \log \frac{w_{\nu}}{z_{\nu}}+\sum_{\mu=1}^{n} \sum_{\nu=1}^{n} x_{\mu} x_{\nu} \log \frac{w_{\mu}-w_{\nu}}{z_{\mu}-z_{\nu}}$

while (2.6) and (2.7) continue to hold without change. Hence (2.5) follows from the Schiffer-Tammi inequality (2.3)

Now we discuss the equality sign in (2.4) and (2.5).

Proposition 2.2. Let $f(z)=\alpha z+\ldots$ be univalent in $\mathbb{D}$ and $f(\mathbb{D}) \subset \mathbb{D}$, $\alpha>0$. Assume $z_{\nu} \in \mathbb{D}, w_{\nu}=f\left(z_{\nu}\right)$, and $x_{\nu} \in \mathbb{R}, x_{1}+\cdots+x_{n}=1$ for $(\nu=1, \ldots, n)$. Suppose that

$$
\sum_{\mu=1}^{n} \sum_{\nu=1}^{n} x_{\mu} x_{\nu} \log \left|\frac{\alpha z_{\mu} z_{\nu}}{w_{\mu} w_{\nu}} \cdot \frac{w_{\mu}-w_{\nu}}{z_{\mu}-z_{\nu}} \cdot \frac{1-w_{\mu} \bar{w}_{\nu}}{1-z_{\mu} \bar{z}_{\nu}}\right|=0 .
$$

Then

$$
\frac{\alpha z}{f(z)} \prod_{\nu=1}^{n}\left(\frac{1-f(z) / w_{\nu}}{1-z / z_{\nu}} \frac{1-\overline{w_{\nu}} f(z)}{1-\overline{z_{\nu}} z}\right)^{x_{\nu}} \equiv 1
$$

provided

$$
\sum_{\nu=1}^{n} x_{\nu} \arg \frac{w_{\nu}}{z_{\nu}}=0
$$


Proof. We use an elementary result. If

$$
\begin{aligned}
& \sum_{\mu=0}^{n} \sum_{\nu=0}^{n} b_{\mu \nu} x_{\mu} x_{\nu} \geq 0 \quad \text { for } x_{0}, \ldots, x_{n} \in \mathbb{R}, \\
& \sum_{\mu=1}^{n} \sum_{\nu=1}^{n} b_{\mu \nu} x_{\mu} x_{\nu}=0
\end{aligned}
$$

then

$$
\sum_{\nu=1}^{n} b_{0 \nu} x_{\nu}=0
$$

The inequality (2.4) of Theorem 2.1 satisfies (2.11) with

$$
\begin{gathered}
b_{00}=\log \frac{1}{\alpha}, \quad b_{0 \nu}=\arg \frac{w_{\nu}}{z_{\nu}}, \quad(\nu=1, \ldots, n), \\
b_{\mu \nu}=\log \left|\frac{\alpha z_{\mu} z_{\nu}}{w_{\mu} w_{\nu}} \cdot \frac{w_{\mu}-w_{\nu}}{z_{\mu}-z_{\nu}} \cdot \frac{1-w_{\mu} \bar{w}_{\nu}}{1-z_{\mu} \bar{z}_{\nu}}\right|, \quad(\mu, \nu=1, \ldots, n) .
\end{gathered}
$$

Moreover, our assumption (2.8) satisfies (2.12). Thus, our assertion (2.10) follows from (2.13).

The same Theorem 2.1 satisfies (2.11) now with $z \in \mathbb{D}$,

$$
\begin{aligned}
& b_{00}=\log \left|\frac{\alpha z^{2}}{f^{2}(z)} f^{\prime}(z) \frac{1-|f(z)|^{2}}{1-|z|^{2}}\right|, \\
& b_{0 \nu}=\log \left|\frac{\alpha z_{\nu} z}{w_{\nu} f(z)} \cdot \frac{f(z)-w_{\nu}}{z-z_{\nu}} \cdot \frac{1-f(z) \bar{w}_{\nu}}{1-z \bar{z}_{\nu}}\right|, \quad(\nu=1, \ldots, n),
\end{aligned}
$$

and $b_{\mu \nu}$ for $(\mu, \nu=1, \ldots, n)$ is defined as before. Now, the assumption (2.8) and the equality (2.13) imply that

$$
\sum_{\nu=1}^{n} x_{\nu} \log \left|\frac{\alpha z_{\nu} z}{w_{\nu} f(z)} \cdot \frac{f(z)-w_{\nu}}{z-z_{\nu}} \cdot \frac{1-f(z) \bar{w}_{\nu}}{1-z \bar{z}_{\nu}}\right| \equiv 0
$$

for $z \in \mathbb{D}$. Finally, the equalities $x_{1}+\cdots+x_{n}=1$ and $f^{\prime}(0)=\alpha$ yield the assertion (2.9).

\section{Remarks.}

1. The method of contour integration used by M. Schiffer and O. Tammi gives no information about the equality sign problem.

2. If there exists a univalent function $f(z)=\alpha z+\ldots$ univalent in $\mathbb{D}$ and $f(\mathbb{D}) \subset \mathbb{D}, \alpha>0$ which satisfies $(2.9)$, then

$$
\prod_{\nu=1}^{n}\left|\frac{\alpha z_{\mu} z_{\nu}}{w_{\mu} w_{\nu}} \cdot \frac{w_{\mu}-w_{\nu}}{z_{\mu}-z_{\nu}} \cdot \frac{1-w_{\mu} \bar{w}_{\nu}}{1-z_{\mu} \bar{z}_{\nu}}\right|^{x_{\nu}}=1, \quad(\mu=1, \ldots, n),
$$

and this implies (2.8). 
Now we deduce the precise form of the extremal function.

Theorem 2.3. Assume $x_{\nu}>0,(\nu=1, \ldots, n), x_{1}+\cdots+x_{n}=1$, the points $z_{\nu} \in \mathbb{T}$ are fixed and $z_{\nu} \neq z_{\mu}$ for $\mu \neq \nu$. Let points $w_{\nu} \in \mathbb{T}$ preserve the same cyclic order in $\mathbb{T}$ as the points $z_{\nu}$ and $w_{\nu} \neq w_{\mu}$ for $\mu \neq \nu$. Suppose that

$$
\sum_{\nu=1}^{n} x_{\nu} \arg z_{\nu}=\sum_{\nu=1}^{n} x_{\nu} \arg w_{\nu}(\bmod 2 \pi) \text {. }
$$

Then there exists $\alpha_{0}=\alpha_{0}\left(w_{1}, \ldots, w_{n}\right)$ so that $0<\alpha_{0} \leq 1$ and $\alpha_{0}=1$ if and only if $w_{\nu}=z_{\nu},(\nu=1, \ldots, n)$. The function $\alpha_{0}\left(w_{1}, \ldots, w_{n}\right)$ depends continuously on $w_{\nu}$ and the functional equation

$$
\frac{f(z)}{\prod_{\nu=1}^{n}\left(1-\overline{w_{\nu}} f(z)\right)^{2 x_{\nu}}}=\frac{\alpha z}{\prod_{\nu=1}^{n}\left(1-\overline{z_{\nu}} z\right)^{2 x_{\nu}}}, \quad z \in \mathbb{D}
$$

has a unique solution $f(z)=\alpha z+\ldots$ which is univalent in $\mathbb{D}$ and $f(\mathbb{D}) \subset \mathbb{D}$ if and only if $0<\alpha \leq \alpha_{0}\left(w_{1}, \ldots, w_{n}\right)$.

Proof. Set the complex vectors $\mathbf{Z}=\left(z_{1}, \ldots, z_{n}\right), \mathbf{W}=\left(w_{1}, \ldots, w_{n}\right)$. We define the function

$$
g(z, \mathbf{Z})=\frac{z}{\prod_{\nu=1}^{n}\left(1-\overline{z_{\nu}} z\right)^{2 x_{\nu}}}, \quad z \in \mathbb{D} .
$$

This function is univalent in $\mathbb{D}$ and $g(\mathbb{D}, \mathbf{Z})$ is a starlike domain. Rewriting this formula for $z=e^{i \theta}$ we obtain

$$
g\left(e^{i \theta}, \mathbf{Z}\right)=\left(\prod_{\nu=1}^{n} \overline{z_{\nu}} x_{\nu}\right) \prod_{\nu=1}^{n}\left(z_{\nu} e^{-i \theta}-2+\overline{z_{\nu}} e^{i \theta}\right)^{-x_{\nu}} .
$$

Putting $z_{\nu}=e^{i \theta_{\nu}}$ we deduce

$$
g\left(e^{i \theta}, \mathbf{Z}\right)=\left(\prod_{\nu=1}^{n}\left(-\frac{\overline{z_{\nu}}}{4}\right)^{x_{\nu}}\right) \prod_{\nu=1}^{n}\left(\sin \frac{\theta-\theta_{\nu}}{2}\right)^{-2 x_{\nu}} .
$$

We set $\Theta_{n}=\sum_{\nu=1}^{n} x_{\nu} \arg \left(-\overline{z_{\nu}}\right)$ and obtain from (2.17) that

$$
\mathbb{C} \backslash g(\mathbb{D}, \mathbf{Z})=\bigcup_{\nu=1}^{n}\left\{r \exp \left(i\left(\Theta_{n}+2 \pi\left(x_{1}+\cdots+x_{\nu}\right)\right)\right): r_{\nu}(\mathbf{Z}) \leq r<\infty\right\},
$$

where $r_{\nu}=r_{\nu}(\mathbf{Z})>0$. We deduce from (2.14) that

$$
\mathbb{C} \backslash g(\mathbb{D}, \mathbf{W})=\bigcup_{\nu=1}^{n}\left\{r \exp \left(i\left(\Theta_{n}+2 \pi\left(x_{1}+\cdots+x_{\nu}\right)\right)\right): r_{\nu}(\mathbf{W}) \leq r<\infty\right\},
$$


with the same value of $\Theta_{n}$. Now define

$$
\alpha_{0}(\mathbf{W})=\min _{\nu} \frac{r_{\nu}(\mathbf{W})}{r_{\nu}(\mathbf{Z})}
$$

with $\mathbf{Z}$ fixed. Equation (2.15) can be written as

$$
g(f(z), \mathbf{W})=\alpha g(z, \mathbf{Z}), \quad z \in \mathbb{D} .
$$

First we assume that Equation (2.15) or (2.21) have a unique solution $f: \mathbb{D} \rightarrow \mathbb{D}$. Therefore,

$$
\alpha g(\mathbb{D}, \mathbf{Z}) \subset g(\mathbb{D}, \mathbf{W}) .
$$

We deduce by (2.18) and (2.19) that

$$
\alpha r_{\nu}(\mathbf{Z}) \leq r_{\nu}(\mathbf{W}), \quad \text { for } \nu=1, \ldots, n
$$

and by (2.20) that $\alpha \leq \alpha_{0}$.

Vice versa, if $\alpha \leq \alpha_{0}$, then (2.23) holds which implies (2.22). This yields that Equation (2.21) has a unique univalent solution.

Finally, if $\alpha_{0} \geq 1$, then $r_{\nu}(\mathbf{W}) \geq r_{\nu}(\mathbf{Z})$ for $\nu=1, \ldots, n$. So, $g(z, \mathbf{Z})=$ $g(z, \mathbf{W})=z+\ldots$ implies that $r_{\nu}(\mathbf{W})=r_{\nu}(\mathbf{Z})$ and $\mathbf{W}=\mathbf{Z}$.

Corollary 2.4. Let $f: \mathbb{D} \rightarrow \mathbb{D}$ and $f(z)=\alpha z+\ldots$ We have equality in the inequality

$$
\sum_{\mu=1}^{n} \sum_{\nu=1}^{n} x_{\mu} x_{\nu} \log \left|\frac{\alpha z_{\mu} z_{\nu}}{f\left(z_{\mu}\right) f\left(z_{\nu}\right)} \cdot \frac{f\left(z_{\mu}\right)-f\left(z_{\nu}\right)}{z_{\mu}-z_{\nu}} \cdot \frac{1-f\left(z_{\mu}\right) \overline{f\left(z_{\nu}\right)}}{1-z_{\mu} \bar{z}_{\nu}}\right| \geq 0
$$

if and only if the extremal function $f$ is the unique solution of (2.15) and $0<\alpha \leq \alpha_{0}(\mathbf{W})$.

The inequality $0<\alpha<1$ yields that the space of the extremal functions for (2.24) depends on $n-1$ real parameters, because, the function $\alpha_{0}(\mathbf{W})$ is continuous and $\alpha_{0}(\mathbf{Z})=1$ by $(2.20)$.

Corollary 2.5. Let $w=f(z)$ be a conformal map of the unit disk into itself, $f(0)=0$, such that for two points $z_{1}, z_{2} \in \mathbb{T}$ the angular limits $w_{1}=f\left(z_{1}\right)$ and $w_{2}=f\left(z_{2}\right)$ are also lie in $\mathbb{T}$. Suppose also that the angular derivatives $f^{\prime}\left(z_{1}\right), f^{\prime}\left(z_{2}\right)$ are finite. If $f^{\prime}(0)=\alpha$, then the inequality

$$
\left|\frac{f\left(z_{1}\right)-f\left(z_{2}\right)}{z_{1}-z_{2}}\right|^{2 t_{1} t_{2}} \geq \frac{1}{\sqrt{\alpha}\left|f^{\prime}\left(z_{1}\right)\right|^{t_{1}^{2}}\left|f^{\prime}\left(z_{2}\right)\right|^{t_{2}^{2}}}
$$

holds and is sharp for all $t_{1}, t_{2} \in(0, \infty)$. If $t_{1}+t_{2}=1$, then for all $\alpha \in$ $\left(0, \alpha_{0}\left(w_{1}, w_{2}\right)\right]$ the extremal map $w=f^{*}(z)$ is the unique solution of the equation

$$
\frac{w w_{1}^{t_{1}} w_{2}^{t_{2}}}{\left(w-w_{1}\right)^{2 t_{1}}\left(w-w_{2}\right)^{2 t_{2}}}=\frac{\alpha z z_{1}^{t_{1}} z_{2}^{t_{2}}}{\left(z-z_{1}\right)^{2 t_{1}}\left(z-z_{2}\right)^{2 t_{2}}} .
$$


Remark. In terms of quadratic differentials the extremal function maps the unit disk $\mathbb{D}$ onto itself minus one or two slits along the trajectories of the differential

$$
\psi(w) d w^{2}=\frac{1}{4 \pi^{2}} \frac{\left(w-e^{i \eta}\right)^{2}\left(w-e^{i \xi}\right)^{2}}{w^{2}\left(w-w_{1}\right)^{2}\left(w-w_{2}\right)^{2}} d w^{2},
$$

where $\xi, \eta$ are calculated by the equations $\xi+\eta=\pi+\arg w_{1}+\arg w_{2}$ and

$$
t_{2}-t_{1}=\frac{e^{i \xi}+e^{i \eta}}{w_{1}-w_{2}}
$$

The latter assertion about the differential we will establish in $\S 4$.

\section{Moduli and extremal partitions.}

We consider in this section a general problem about the extremal partition of the unit disk and deduce the properties of the weighted sum of the reduced moduli of digons and circular domains.

Let $\mathbb{D}$ be the unit disk and $\mathbb{D}^{\prime}=\mathbb{D} \backslash\{0\}$. On the boundary $\mathbb{T}$ of $\mathbb{D}$ we fix two points $\zeta_{1}=e^{i \theta_{1}}, \zeta_{2}=e^{i \theta_{2}}, 0 \leq \theta_{1}<\theta_{2}<2 \pi$.

1. The first problem about the extremal partition we formulate as follows. Set a nonzero weight vector $\left(t_{1}, t_{2}\right)$ with nonnegative coordinates. We construct two intervals $\gamma_{1}=\left(0, e^{i \theta_{1}}\right)$ and $\gamma_{2}=\left(0, e^{i \theta_{2}}\right)$ in $\mathbb{D}^{\prime}$. These curve system $\left\{\gamma_{1}, \gamma_{2}\right\}$ we call admissible in analogy with the terminology of [15]. Now we consider the pairs of non-overlapping digons $\left(D_{1}, D_{2}\right)$ on $\mathbb{D}^{\prime}$ associated with the curve system $\left\{\gamma_{1}, \gamma_{2}\right\}$, i.e., the digon $D_{j}$ has its vertices at $0, e^{i \theta_{j}}, j=1,2$. We require the digons $D_{1}$ and $D_{2}$ to be conformal at the vertices and to satisfy the condition of compatibility of angles and weights, i.e., $\varphi_{0}^{(j)}=\frac{2 \pi t_{j}}{t_{1}+t_{2}}$ is the inner angle of the digon $D_{j}$ at the origin and $\varphi_{\zeta_{j}}^{(j)}=\pi$ is the inner angle of the digon $D_{j}$ at the boundary point $\zeta_{j}, j=1,2$. The reduced moduli $m\left(D_{j}, 0, \zeta_{j}\right)$ exist. We call the system of defined digons the admissible system of digons.

The results obtained in $[\mathbf{2}, \mathbf{4}, \mathbf{6}, \mathbf{7}]$ (see also [14]) imply that any collection of non-overlapping admissible digons associated with the curve system $\left(\gamma_{1}, \gamma_{2}\right)$ and the condition of compatibility of angles and weights satisfies the following inequality

$$
t_{1}^{2} m\left(D_{1}, 0, e^{i \theta_{1}}\right)+t_{2}^{2} m\left(D_{2}, 0, e^{i \theta_{2}}\right) \geq t_{1}^{2} m\left(D_{1}^{*}, 0, e^{i \theta_{1}}\right)+t_{2}^{2} m\left(D_{2}^{*}, 0, e^{i \theta_{2}}\right)
$$

for a fixed weight vector $\left(t_{1}, t_{2}\right)$ with the equality sign only for $D_{j}=D_{j}^{*}$. We denote this minimum by $\mathcal{M}_{1}\left(t_{1}, t_{2}, 0, e^{i \theta_{1}}, e^{i \theta_{2}}\right)$ showing its dependence on parameters. 
Each $D_{j}^{*}$ is a strip domain in the trajectory structure of a unique quadratic differential

$$
\varphi(z) d z^{2}=A \frac{\left(z-e^{i \gamma}\right)^{2}\left(z-e^{i \beta}\right)^{2}}{z^{2}\left(z-e^{i \theta_{1}}\right)^{2}\left(z-e^{i \theta_{2}}\right)^{2}} d z^{2}, \quad A>0,
$$

associated with the problem of the extremal partition (see $[\mathbf{2}, \mathbf{6}, \mathbf{7}, \mathbf{1 4}]$ for the details). The factor $A$ is positive because of strip domains and the local trajectory structure close to the origin $\left(\varphi(z)=\frac{A}{z^{2}}(1+\ldots)\right)$. Here $A, \gamma$, and $\beta$ are functions of $t_{1}, t_{2}$. For $D_{j}^{*}$ there is a conformal map $g_{j}(z), z \in D_{j}^{*}$ satisfying the differential equation

$$
t_{j}^{2}\left(\frac{g_{j}^{\prime}(z)}{g_{j}(z)}\right)^{2}=\pi^{2} \varphi(z),
$$

that maps $D_{j}^{*}$ onto the digon $\mathbb{H}^{+}=\{z: \operatorname{Im} z>0\}$ with the vertices at 0 and $\infty$.

The critical trajectories of $\varphi(z) d z^{2}$ split $\mathbb{D}^{\prime}$ into at most two strip domains $\left\{D_{j}^{*}\right\}$ associated with the admissible system (one of $D_{j}^{*}$ can degenerate).

Theorem 3.1. Let $t_{1}, t_{1} \in(0, \infty), 0 \leq \theta_{1}<\theta_{2}<2 \pi, \theta_{2}-\theta_{1} \leq \pi$. Then

$$
\begin{aligned}
& m\left(D_{1}^{*}, 0, e^{i \theta_{1}}\right)=\frac{t_{2}}{\pi t_{1}} \log \frac{1}{2\left|\sin \frac{\theta_{1}-\theta_{2}}{2}\right|}, \\
& m\left(D_{2}^{*}, 0, e^{i \theta_{2}}\right)=\frac{t_{1}}{\pi t_{2}} \log \frac{1}{2\left|\sin \frac{\theta_{1}-\theta_{2}}{2}\right|} .
\end{aligned}
$$

In the differential (3.2) we have

$$
\begin{gathered}
A=\frac{\left(t_{1}+t_{2}\right)^{2}}{4 \pi^{2}}, \quad \beta=\sin ^{-1}\left(\frac{t_{2}-t_{1}}{t_{2}+t_{1}} \sin \frac{\theta_{1}-\theta_{2}}{2}\right)+\frac{\theta_{1}+\theta_{2}}{2}, \\
\gamma=\pi-\beta+\theta_{1}+\theta_{2},
\end{gathered}
$$

and two critical trajectories of the differential $\varphi(z) d z^{2}$ starting from the origin under the angle $\varphi_{0}^{(1)}=\frac{2 \pi t_{1}}{t_{1}+t_{2}}$ end at the points $e^{i \beta}$ and $e^{i \gamma}$.

Proof. First we observe that the unit circle $\mathbb{T}$ is a trajectory of the differential (3.2). Therefore, $-\varphi\left(e^{i \theta}\right) e^{2 i \theta} d \theta^{2}>0$ at all regular points of $\mathbb{T}$. This implies that $\beta+\gamma=\pi+\theta_{1}+\theta_{2}$.

Rotating the configuration by $z \rightarrow z e^{i \frac{\theta_{1}+\theta_{2}}{2}}$ without loss of generality we assume that $\theta_{2} \in(0, \pi / 2]$ and $\theta_{1}=-\theta_{2}$.

Close to the origin the map $g_{1}(z)$ has the expansion $g_{1}=z^{\pi / \varphi_{0}^{(1)}}\left(c_{1}+\ldots\right)$. Tending in (3.3) $z \rightarrow 0$ for $j=1$ we obtain $\frac{t_{1}^{2}}{\left(\varphi_{0}^{(1)}\right)^{2}}=A$. Close to the origin the map $g_{2}(z)$ has the expansion $g_{2}=z^{\pi / \varphi_{0}^{(2)}}\left(c_{2}+\ldots\right)$. Tending in (3.3) $z \rightarrow 0$ for $j=2$ we obtain $\frac{t_{2}^{2}}{\left(\varphi_{0}^{(2)}\right)^{2}}=A$. We calculate $A=\frac{\left(t_{1}+t_{2}\right)^{2}}{4 \pi^{2}}$ and see 
that the extremal domains $D_{1}^{*}, D_{2}^{*}$ satisfy the condition of compatibility of weights and angles. Close to the point $e^{i \theta_{1}}$ the map $g_{1}(z)$ has the expansion $g_{1}=\left(z-e^{i \theta_{1}}\right)\left(d_{1}+\ldots\right)$. Tending in (3.3) $z \rightarrow e^{i \theta_{1}}$ for $j=1$ we obtain $\frac{2 t_{1}}{t_{1}+t_{2}}=1-\frac{\sin \beta}{\sin \theta_{1}}$ or $\beta=\sin ^{-1}\left(\frac{t_{2}-t_{1}}{t_{2}+t_{1}} \sin \theta_{1}\right)$.

Suppose $j=1$. We take the square root in (3.3) and integrate it putting the branch of the root so that $g(0)=\infty$ and $g\left(e^{i \theta_{2}}\right)=0$. We normalize the function $g(z)$ so that the part of the the boundary of $D_{1}^{*}$ lying on the unit circle is mapped into the real axis. Therefore, normalizing the imaginary constant in the logarithm and exponentiating we obtain

$$
g_{1}(z)=\frac{\left(z-e^{i \theta_{1}}\right)\left(z-e^{-i \theta_{1}}\right)^{t_{2} / t_{1}}}{z^{\frac{t_{1}+t_{2}}{2 t_{1}}}} e^{i\left(\theta_{1} \frac{t_{2}-t_{1}}{2 t_{1}}-\frac{\pi}{2} \frac{t_{1}+t_{2}}{t_{1}}\right)} .
$$

Suppose $j=2$. By analogy to the previous case we obtain

$$
g_{2}(z)=\frac{\left(z-e^{i \theta_{1}}\right)^{t_{1} / t_{2}}\left(z-e^{-i \theta_{1}}\right)}{z^{\frac{t_{1}+t_{2}}{2 t_{2}}}} e^{i\left(\theta_{1} \frac{t_{2}-t_{1}}{2 t_{2}}-\frac{\pi}{2} \frac{t_{1}+t_{2}}{t_{2}}\right)} .
$$

The reduced modulus of the digon $\mathbb{H}^{+}$with respect to its vertices at 0 and $\infty$ is equal to zero. Therefore, calculating the derivatives of the maps $g_{1}$ and $g_{2}$ and using the formulas (1.3), (1.4) on the change of the reduced moduli under conformal map, we obtain the values of the reduced moduli $m\left(D_{1}^{*}, 0, e^{i \theta_{1}}\right)$, $m\left(D_{2}^{*}, 0, e^{i \theta_{2}}\right)$ asserted in Theorem 3.1. This finishes the proof.

Remark. The case $\theta_{2}-\theta_{1}>\pi$ can be treated similarly and the moduli in Theorem 3.1 are the same interchanging $\gamma \leftrightarrow \beta$.

2. Now we consider another problem about the extremal partition of $\mathbb{D}^{\prime}$. In $\mathbb{D}^{\prime}$ with two fixed boundary points $\zeta_{1}, \zeta_{2}$ we set the admissible homotopy curve system $\gamma^{(m)}=\left(\gamma_{1}, \gamma_{2}^{(m)}\right), m=1,2$. For definiteness we assume $0 \leq$ $\theta_{1}<\theta_{2}<2 \pi, \theta_{2}-\theta_{1} \leq \pi$. The case $\theta_{2}-\theta_{1}>\pi$ is symmetric as it was mentioned in the remark after Theorem 3.1. The curve $\gamma_{1}$ is a circle $\{z$ : $|z|=\varepsilon\}$ for a sufficiently small $\varepsilon \cdot \gamma_{2}^{(m)}$ is the segment $\left[e^{i \theta_{1}}, e^{i \theta_{2}}\right]$ for $m=1$ or the broken line with the legs $\left[e^{i \theta_{1}},-\frac{1}{2} e^{i\left(\theta_{2}-\theta_{1}\right) / 2}\right]$ and $\left[-\frac{1}{2} e^{i\left(\theta_{2}-\theta_{1}\right) / 2}, e^{i \theta_{2}}\right]$ for $m=2$.

We consider the pairs of non-overlapping domains $\left(D_{1}, D_{2}\right)$ in $\mathbb{D}^{\prime}$ associated with the curve system $\gamma^{(m)}$, i.e., $D_{1}$ is a simply connected hyperbolic domain, $0 \in D_{1}$, and $D_{2}$ is a digon with the vertices at $\zeta_{1}, \zeta_{2}$. We require the digon $D_{2}$ to be conformal at its vertices and to satisfy the condition of compatibility of angles and weights, i.e., $\varphi_{\zeta_{1}}=\varphi_{\zeta_{2}}=\pi$ are the inner angles of the digon $D_{2}$. Therefore, the reduced modulus $m\left(D_{2}, \zeta_{1}, \zeta_{2}\right)$ exists. We call the system $\mathfrak{D}$ of such $\left(D_{1}, D_{2}\right)$ the admissible system of domains.

We set a weight nonzero vector $\left(t_{1}, t_{2}\right)$ with nonnegative coordinates. The results in $[\mathbf{2}, \mathbf{7}]$ (see also [14]) imply that any collection of non-overlapping 
admissible domains associated with the curve system $\gamma^{(m)}=\left(\gamma_{1}, \gamma_{2}^{(m)}\right)$ and the condition of compatibility of angles and weights for digon satisfies the following inequality

$$
t_{1}^{2} m\left(D_{1}, 0\right)-t_{2}^{2} m\left(D_{2}, \zeta_{1}, \zeta_{2}\right) \leq t_{1}^{2} m\left(D_{1}^{*}, 0\right)-t_{2}^{2} m\left(D_{2}^{*}, \zeta_{1}, \zeta_{2}\right)
$$

for the weight vector $\left(t_{1}, t_{2}\right)$ fixed with the equality sign only for $D_{j}=D_{j}^{*}$, $j=1,2$. We denote this minimum by $\mathcal{M}_{2}^{(m)}\left(t_{1}, t_{2}, 0, e^{i \theta_{1}}, e^{i \theta_{2}}\right)$ displaying its dependence on parameters.

Rotating the configuration by $z \rightarrow z e^{i \frac{\theta_{1}+\theta_{2}}{2}}$ we assume that $\theta_{2} \in(0, \pi / 2]$ and $\theta_{1}=-\theta_{2}$.

We consider $m=1$. Under the conformal transformation

$$
z \rightarrow \frac{z+1 / z-2 \cos \theta_{2}}{2-2 \cos \theta_{2}}
$$

the extremal configuration $\left(D_{1}^{*}, D_{2}^{*}\right)$ is mapped onto the extremal configuration $\left(B_{1}^{*}, B_{2}^{*}\right)$ in the following problem on the extremal partition which is given in Theorems 3.2-3.3.

Let $S_{0}=\mathbb{C} \backslash\{0, a\}$ be the twice-punctured complex plane. Assume $a \neq 1$ and the case $a=1$ is the limiting case as $a \rightarrow 1$.

We consider on $S_{0}$ the admissible system $\left(\gamma_{1}, \gamma_{2}\right)$ where $\gamma_{1}=\{z:|z|=$ $1 / \varepsilon\}$ and $\gamma_{2}=\{z:|z-a|=a\}$ so that $\varepsilon$ is sufficiently small such that $\gamma_{1}$ and $\gamma_{2}$ do not intersect. With this admissible system we associate admissible pairs $\left(B_{1}, B_{2}\right)$ of circular domains $B_{1}$ in $S_{0}$ so that $\infty \in B_{1}$ and digons $B_{2}$ with the vertices at 0 . Each digon $B_{2}$ has its inner angles $\pi$ at 0 (the condition of compatibility).

Let $\mathfrak{B}$ be the set of all pairs $\left(B_{1}, B_{2}\right)$ each of which consists of a circular domain and a digon associated with the admissible system $\left(\gamma_{1}, \gamma_{2}\right)$. Then the problem of the extremal partition of $S_{0}$ consists of maximizing the sum $t_{1}^{2} m\left(B_{1}, \infty\right)-t_{2}^{2} m\left(B_{2}, 0,0\right)$ as $\left(B_{1}, B_{2}\right) \in \mathfrak{B}$. Without loss of generality, we assume $t_{1}=t, t_{2}=1, t \in[0, \infty)$, and the maximum of this sum we denote by $M(t, a)$. There is a unique pair $\left(B_{1}^{*}, B_{2}^{*}\right)$ which is extremal in this problem. $B_{1}^{*}$ is the circular domain and $B_{2}^{*}$ is the strip domain in the trajectory structure of the differential

$$
\phi(z) d z^{2}=-A \frac{(z-c) d z^{2}}{z^{2}(z-a)}, \quad A>0, \quad c \leq 0 .
$$

Here $A$ and $c$ are functions of $t$. If $t=0$, then $B_{1}^{*}=\emptyset$ and $B_{2}^{*}=\mathbb{C} \backslash(-\infty, a]$ is the digon with two vertices with the same support 0 . In this case $M(0, a)=$ $\frac{2}{\pi} \log 4 a$. If $t \rightarrow \infty$, then $D_{1}^{*}=\mathbb{C} \backslash[0, a]$. In this case $M(\infty, a)=\frac{1}{2 \pi} \log 4 / a$. 
Theorem 3.2. Let $0<t<\infty$. Then

$$
\begin{aligned}
m\left(B_{1}^{*}, \infty\right) & =\frac{1}{2 \pi} \log \frac{4 t^{2}}{a\left(1+t^{2}\right)}-\frac{1}{\pi t}\left(\frac{\pi}{2}-\tan ^{-1} \frac{1}{t}\right), \\
m\left(B_{2}^{*}, 0,0\right) & =\frac{2}{\pi} \log \frac{4 a}{1+t^{2}}+\frac{4 t}{\pi}\left(\frac{\pi}{2}-\tan ^{-1} \frac{1}{t}\right) .
\end{aligned}
$$

The constants for the differential $\phi$ are $A=t / 4 \pi^{2}, c=-a / t^{2}$.

Proof. We consider the mapping $u=u(z)$ whose inverse is

$$
z=c \frac{a+1+(a-1) \cos u}{(c+1)+(c-1) \cos u},
$$

and obtain the representation of the differential $\phi$ in terms of the parameter $u$ in regular points

$$
\begin{aligned}
\phi(z) d z^{2} & =Q(u) d u^{2} \\
& =\frac{4 A c(a-c)^{2}(1+\cos u)^{2}}{((c+1)+(c-1) \cos u)^{2}((a+1)+(a-1) \cos u)^{2}} d u^{2} .
\end{aligned}
$$

Here

$$
\left|\frac{a+1}{a-1}\right|>1, \quad \text { and } \quad\left|\frac{c+1}{c-1}\right| \leq 1 .
$$

Now we study the trajectory structure of this quadratic differential that is a complete square of a linear one. The differential $Q(u) d u^{2}$ has zeros of order 4 at the points $\pi+2 \pi k$ which are the images of $c$ under the mapping $u(z)$. Furthermore, $u(0)= \pm \eta_{k}$, so that $\operatorname{Re} \eta_{0}=0$ in case $a<1$ or $\operatorname{Re} \eta_{0}=\pi$ in case $a>1$, and

$$
\eta_{k}=\cos ^{-1} \frac{1+a}{1-a}, \quad k=1,2, \ldots, n, \ldots
$$

For definiteness, assume now $a<1$. Then $u(\infty)=\theta_{k}=\cos ^{-1}(1+c) /(1-c)$, $\theta_{0} \in(0, \pi)$ and $\theta_{k}, \eta_{k}$ are the poles of second order. The points $u(a)=2 \pi k$ are regular for this differential.

Consider a fixed branch of the function $u(z)$ which maps $\overline{\mathbb{C}} \backslash[c, a]$ onto the strip $0<\operatorname{Re} u<\pi$. The circular domain $B_{1}^{u}=u\left(D_{1}^{*}\right)$ is bounded by the critical trajectory of $Q(u) d u^{2}$ starting and ending at $\pi$ enclosing the real point $\theta_{0}$. The strip domain $B_{2}^{u}=u\left(D_{2}^{*}\right)$ is bounded by the same trajectory, the imaginary axis, and the straight line $\operatorname{Re} u=\pi$.

Let $\zeta_{j}(u), j=1,2$ be conformal mappings from the domains $B_{j}^{u}$ onto the unit disk $\mathbb{D}$ and the digon $\mathbb{C} \backslash[0, \infty)$ respectively, such that $\zeta_{1}\left(\theta_{0}\right)=0$ and $\zeta_{2}\left(\eta_{0}\right)=0, \zeta_{2}\left(-\eta_{0}\right)=\infty$. These functions satisfy in the domains $B_{1}^{u}$ the differential equation

$$
t \frac{d \zeta_{1}(u)}{\zeta_{1}(u)}=2 \pi \sqrt{-Q(u)} d u
$$


and in the domains $B_{2}^{u}$ the differential equation

$$
\frac{d \zeta_{2}(u)}{\zeta_{2}(u)}=2 \pi \sqrt{Q(u)} d u
$$

or in terms of the parameter $z$

$$
\begin{gathered}
t\left(\frac{d \zeta_{1}(u(z))}{\zeta_{1}(u(z))}\right)^{2}=-4 \pi^{2} \phi(z) d z^{2}, \\
\left(\frac{d \zeta_{2}(u(z))}{\zeta_{2}(u(z))}\right)^{2}=4 \pi^{2} \phi(z) d z^{2} .
\end{gathered}
$$

Letting $z \rightarrow \infty$ in (3.12) in the case of $j=1$ or $z \rightarrow 0$ in (3.13) in the case of $j=2$, we obtain $A=t / 4 \pi^{2}$ and $c=-a / t^{2}$.

Now we calculate the reduced modulus of the circular domain. The part $\left[\theta_{0}+\delta, \pi\right]$ of the orthogonal trajectory of the differential $Q(u) d u^{2}$ for sufficiently small $\delta$ has a preimage $\left[-1 / \varepsilon_{1}, c\right]$ under the mapping $u(z)$. From (3.8) we derive

$$
\delta=\frac{\sqrt{-c}(a-c)}{1-c} \varepsilon_{1}+O\left(\varepsilon_{1}^{2}\right) .
$$

These two segments have the image in the $\zeta$-plane $\left[\varepsilon e^{i \beta}, e^{i \beta}\right]$. Without loss of generality assume $\beta=0$.

Let $z=f_{1}(\zeta)=A_{-1} / \zeta+A_{0}+A_{1} \zeta+\ldots$ be a function from $\mathbb{D}$ onto $B_{1}^{*}$. Then the reduced modulus of $B_{1}^{*}$ can be calculated as $m\left(B_{1}^{*}, \infty\right)=$ $\frac{1}{2 \pi} \log 1 /\left|A_{1}\right|$. We have directly from (3.9)

$$
\begin{aligned}
\sqrt{-Q(u)} & =2 \sqrt{-c A}\left(\frac{1}{c+1+(c-1) \cos u}-\frac{1}{a+1+(a-1) \cos u}\right) \\
& = \pm \frac{1}{2 \pi} \cdot \frac{d}{d u}\left(t \log \frac{t \tan \frac{u}{2}-\sqrt{a}}{t \tan \frac{u}{2}+\sqrt{a}}-2 \tan ^{-1} \frac{\tan \frac{u}{2}}{\sqrt{a}}\right) .
\end{aligned}
$$

Let us choose the branch of the root such that we have $(+)$ in front of the previous expression. Moreover, $\tan \left(\theta_{0} / 2\right)=\sqrt{-c}=\sqrt{a} / t$. Integrating (3.10) along the described segments we derive

$$
\begin{aligned}
\varepsilon & =\frac{t \tan \frac{\theta_{0}+\delta}{2}-\sqrt{a}}{t \tan \frac{\theta_{0}+\delta}{2}+\sqrt{a}} \cdot \exp \left(\frac{2}{t}\left(\frac{\pi}{2}-\tan ^{-1} \frac{\tan \frac{\theta_{0}+\delta}{2}}{\sqrt{a}}\right)\right) \\
& =\frac{\left(a+t^{2}\right)}{4 t \sqrt{a}} \exp \left(\frac{2}{t}\left(\frac{\pi}{2}-\tan ^{-1} \frac{1}{t}\right)\right) \cdot \delta+O\left(\delta^{2}\right)
\end{aligned}
$$

and, finally, using (3.14) we obtain

$$
A_{1}=\frac{a\left(1+t^{2}\right)}{4 t^{2}} \exp \left(\frac{2}{t}\left(\frac{\pi}{2}-\tan ^{-1} \frac{1}{t}\right)\right) .
$$


Then the modulus $m\left(B_{1}^{*}, \infty\right)$ has the form stated in Theorem 3.2.

Next we calculate the reduced modulus of the digon $B_{2}^{*}$ with respect to its two vertices with the same support 0 . For this we consider the strip domain $B_{2}^{u}$ in the $u$-plane and the segment of the imaginary axis $\left[0, \eta_{0}-i \delta\right]$ that belongs to the critical trajectory of the differential $Q(u) d u^{2}$. It has a preimage $\left[\varepsilon_{1}, a\right]$ in the $z$-plane under the mapping $u(z)$. We calculate from (3.8) that

$$
\delta=\frac{c-a}{c \sqrt{a}(a-1)} \varepsilon_{1}+O\left(\varepsilon_{1}^{2}\right)
$$

For these two segments there is an image in the $\zeta$-plane $[1,1 / \varepsilon]$ that belongs to the boundary of the digon $\mathbb{C} \backslash[0, \infty)$ which is the image of the domain $B_{2}^{*}$ under the map $\zeta_{2}(u(z))$. We find that the lengths of the segments $[\varepsilon, 1]$ and $[1,1 / \varepsilon]$ are equal in the metric $|d \zeta| /|\zeta|$ and, therefore, $1=\zeta_{2}(0)$. Let $z=$ $f_{2}(\zeta)=B_{-1} / \zeta+B_{0}+B_{1} \zeta+\ldots$ be the conformal map from $\mathbb{C} \backslash[0, \infty)$ onto $B_{2}^{*}$. Then the reduced modulus of $B_{2}^{*}$ turns out to be $m\left(B_{2}^{*}, 0,0\right)=\frac{2}{\pi} \log \left|B_{-1}\right|$. Here we understand this derivative as one of the angular derivatives in $B_{2}^{*}$.

By (3.15) we have

$$
\sqrt{Q(u)}= \pm \frac{i}{2 \pi} \cdot \frac{d}{d u}\left(t \log \frac{t \tan \frac{u}{2}-\sqrt{a}}{t \tan \frac{u}{2}+\sqrt{a}}-2 \tan ^{-1} \frac{\tan \frac{u}{2}}{\sqrt{a}}\right) .
$$

Again we choose the branch of the root such that we have $(+)$ in front of the right-hand side of (3.17). We have $\tan \left(\eta_{0} / 2\right)=i \sqrt{a}$. We rewrite Equation (3.11) as

$$
\frac{d \zeta}{\zeta}=i \frac{d}{d u}\left(t \log \frac{t \tan \frac{u}{2}-\sqrt{a}}{t \tan \frac{u}{2}+\sqrt{a}}-2 \tan ^{-1} \frac{\tan \frac{u}{2}}{\sqrt{a}}\right) d u .
$$

Since we use the complex tangent, we better transfer the right-hand side using the transform identity

$$
\tan ^{-1} w=\frac{1}{2 i} \log \frac{1+i w}{1-i w} .
$$

Then,

$$
\frac{d \zeta}{\zeta}=\frac{d}{d u}\left(-2 t \tan ^{-1} \frac{i \sqrt{a}}{t \tan \frac{u}{2}}-\log \frac{1+i \frac{\tan \frac{u}{2}}{\sqrt{a}}}{1-i \frac{\tan \frac{u}{2}}{\sqrt{a}}}\right)
$$


Integrating (3.18) along the segment $[1,1 / \varepsilon]$ in the left-hand side and along the vertical segment $\left[0, \eta_{0}-i \delta\right], \operatorname{Im} \eta_{0}>0$, in the right-hand side, we deduce

$$
\begin{aligned}
\varepsilon & =\frac{1+i \frac{\tan \frac{\eta_{0}-i \delta}{2}}{\sqrt{a}}}{1-i \frac{\tan \frac{\eta_{0}-i \delta}{2}}{\sqrt{a}}} \cdot \exp \left(2 t\left(\tan ^{-1} \frac{i \sqrt{a}}{t \tan \frac{\eta_{0}-i \delta}{2}}-\frac{\pi}{2}\right)\right) \\
& =\frac{1-a}{4 \sqrt{a}} \exp \left(2 t\left(\tan ^{-1} \frac{1}{t}-\frac{\pi}{2}\right)\right) \delta+O\left(\delta^{2}\right) .
\end{aligned}
$$

Finally, using (3.14) and substituting $c=-a / t^{2}$ we obtain

$$
\left|B_{1}\right|=\frac{4 a}{1+t^{2}} \exp \left(2 t\left(\frac{\pi}{2}-\tan ^{-1} \frac{1}{t}\right)\right) .
$$

This leads to the expression in Theorem 3.2. The case $a>1$ can be obtained by applying the mapping $w=k z$, where $k>1 / a$. This leads to the same expressions.

Let $S_{1}=\mathbb{C} \backslash\left\{c_{1}, 0, a_{1}\right\}, a_{1}>0, c_{1}<0$, be the thrice-punctured complex plane. We consider on $S_{1}$ the admissible system $\left(\gamma_{1}, \gamma_{2}\right)$ where $\gamma_{1}=\{z$ : $|z|=1 / \varepsilon\}$ and $\gamma_{2}=\left\{z:\left|z-a_{1}-\varepsilon\right|=a_{1}+\varepsilon\right\}$, so that $\varepsilon$ is sufficiently small. Let $\mathfrak{B}$ be the set of all pairs $\left(B_{1}, B_{2}\right)$ consisting of a circular domain and a digon associated with the admissible system $\left(\gamma_{1}, \gamma_{2}\right)$. The condition of compatibility for the digon $B_{2}$ is the same as before. The inner angles of $D_{2}$ at the vertices over 0 are $\pi$. Then the problem of extremal partition of $S_{1}$ consists of finding the maximum of the sum $t_{1}^{2} m\left(B_{1}, \infty\right)-t_{2}^{2} m\left(B_{2}, 0,0\right)$ as $\left(B_{1}, B_{2}\right) \in \mathfrak{B}$. Without loss of generality, assume $t_{1}=t, t_{2}=1, t \in[0, \infty)$, and the maximum of this sum we denote by $M\left(t, c_{1}, a_{1}\right)$. There is a unique pair $\left(B_{1}^{*}, B_{2}^{*}\right)$ that is extremal in this problem. $B_{1}^{*}$ is the circular domain and $B_{2}^{*}$ is the strip domain in the trajectory structure of the differential

$$
\psi(z) d z^{2}=-A \frac{(z-b)^{2} d z^{2}}{z^{2}\left(z-a_{1}\right)\left(z-c_{1}\right)}, \quad A>0, \quad b \leq 0 .
$$

Here $A$ and $b$ are functions of $t$. For $t \in\left[0, \sqrt{\frac{a_{1}}{-c_{1}}}\right]$ the problem can be reduced to that in the previous case with $a=a_{1}$. If $t \rightarrow \infty$, then $B_{1}^{*}=$ $\mathbb{C} \backslash\left[c_{1}, a_{1}\right]$. In this case $M\left(\infty, c_{1}, a_{1}\right)=\frac{1}{2 \pi} \log 4 /\left(a_{1}-c_{1}\right)$.

Theorem 3.3. Let $\sqrt{\frac{a_{1}}{-c_{1}}} \leq t<\infty$. Then

$$
\begin{aligned}
m\left(B_{1}^{*}, \infty\right) & =\frac{1}{2 \pi} \log \frac{4}{a_{1}-c_{1}}-\frac{1}{\pi t}\left(\frac{\pi}{2}-\tan ^{-1} \sqrt{\frac{-c_{1}}{a_{1}}}\right), \\
m\left(B_{2}^{*}, 0,0\right) & =\frac{2}{\pi} \log \frac{-4 a_{1} c_{1}}{a_{1}-c_{1}}+\frac{4 t}{\pi}\left(\frac{\pi}{2}-\tan ^{-1} \sqrt{\frac{-c_{1}}{a_{1}}}\right) .
\end{aligned}
$$

The constants for the differential $\psi$ are $A=t / 4 \pi^{2}, b=-\sqrt{-a_{1} c_{1}} / t$. 
The proof is similar to that for Theorem 3.2

By Theorems 3.2-3.3 and the conformal transformation (3.7) using the formulas of the change of the reduced moduli ( problem about $\mathcal{M}_{2}^{(1)}\left(t, 1,0, \zeta_{1}, \zeta_{2}\right)$ for all $\theta_{1}, \theta_{2}$ such that $\left|\theta_{2}-\theta_{1}\right| \leq \pi$ and for

$$
0<t \leq \sqrt{\frac{1-\cos \frac{\theta_{2}-\theta_{1}}{2}}{1+\cos \frac{\theta_{2}-\theta_{1}}{2}}}=\tan \frac{\theta_{2}-\theta_{1}}{4}
$$

the reduced moduli are expressed as

$$
\begin{aligned}
m\left(D_{1}^{*}, 0\right) & =\frac{1}{2 \pi} \log \frac{2 t^{2}}{\left(1+t^{2}\right)\left(1-\cos \frac{\theta_{2}-\theta_{1}}{2}\right)}-\frac{1}{\pi t}\left(\frac{\pi}{2}-\tan ^{-1} \frac{1}{t}\right), \\
m\left(D_{2}^{*}, \zeta_{1}, \zeta_{2}\right) & =\frac{2}{\pi} \log \frac{4}{1+t^{2}} \frac{1-\cos \frac{\theta_{2}-\theta_{1}}{2}}{\sin \frac{\theta_{2}-\theta_{1}}{2}}+\frac{4 t}{\pi}\left(\frac{\pi}{2}-\tan ^{-1} \frac{1}{t}\right) .
\end{aligned}
$$

For

$$
t>\tan \frac{\theta_{2}-\theta_{1}}{4}
$$

the reduced moduli are expressed as

$$
\begin{aligned}
m\left(D_{1}^{*}, 0\right) & =-\frac{\theta_{2}-\theta_{1}}{4 \pi t}, \\
m\left(D_{2}^{*}, \zeta_{1}, \zeta_{2}\right) & =\frac{2}{\pi} \log \left(2 \sin \frac{\theta_{2}-\theta_{1}}{2}\right)+\frac{t}{\pi}\left(\theta_{2}-\theta_{1}\right) .
\end{aligned}
$$

In the problem about $\mathcal{M}_{2}^{(2)}\left(t, 1,0, \zeta_{1}, \zeta_{2}\right)$ we use the conformal transformation

$$
z \rightarrow-\frac{z+1 / z-2 \cos \theta_{2}}{2+2 \cos \theta_{2}}
$$

instead of (3.7) and deduce analogously for

$$
0<t \leq \tan ^{-1} \frac{\theta_{2}-\theta_{1}}{4}
$$

the reduced moduli are expressed as

$$
\begin{aligned}
m\left(D_{1}^{*}, 0\right) & =\frac{1}{2 \pi} \log \frac{2 t^{2}}{\left(1+t^{2}\right)\left(1+\cos \frac{\theta_{2}-\theta_{1}}{2}\right)}-\frac{1}{\pi t}\left(\frac{\pi}{2}-\tan ^{-1} \frac{1}{t}\right), \\
m\left(D_{2}^{*}, \zeta_{1}, \zeta_{2}\right) & =\frac{2}{\pi} \log \frac{4}{1+t^{2}} \frac{1+\cos \frac{\theta_{2}-\theta_{1}}{2}}{\sin \frac{\theta_{2}-\theta_{1}}{2}}+\frac{4 t}{\pi}\left(\frac{\pi}{2}-\tan ^{-1} \frac{1}{t}\right) .
\end{aligned}
$$

For

$$
t>\tan ^{-1} \frac{\theta_{2}-\theta_{1}}{4}
$$


the reduced moduli are expressed as

$$
\begin{aligned}
m\left(D_{1}^{*}, 0\right) & =\frac{1}{\pi t}\left(\frac{\theta_{2}-\theta_{1}}{4}-\frac{\pi}{2}\right), \\
m\left(D_{2}^{*}, \zeta_{1}, \zeta_{2}\right) & =\frac{2}{\pi} \log \left(2 \sin \frac{\theta_{2}-\theta_{1}}{2}\right)+\frac{t}{\pi}\left(2 \pi-\left(\theta_{2}-\theta_{1}\right)\right) .
\end{aligned}
$$

The limiting case $t \rightarrow \infty$ yields $\mathcal{M}_{2}^{(j)}\left(\infty, 1,0, \zeta_{1}, \zeta_{2}\right)=0 ; t \rightarrow 0$ yields

$$
\mathcal{M}_{2}^{(j)}\left(0,1,0, \zeta_{1}, \zeta_{2}\right)=\frac{2}{\pi} \log 4 \frac{1-(-1)^{j} \cos \frac{\theta_{2}-\theta_{1}}{2}}{\sin \frac{\theta_{2}-\theta_{1}}{2}}, \quad j=1,2 .
$$

\section{Extremal problems.}

In this section we apply the results about the extremal partition to finding the sharp estimates of the functionals in the class of bounded univalent functions $f: \mathbb{D} \rightarrow \mathbb{D}, f(0)=0$ with the finite angular limits and derivatives. We start with functions that have the angular limits $f\left(\zeta_{1}\right), f\left(\zeta_{2}\right) \in \mathbb{T}$ at the points $\zeta_{1}, \zeta_{2} \in \mathbb{T}$ and the finite nonzero angular derivatives $f^{\prime}\left(\zeta_{1}\right), f^{\prime}\left(\zeta_{2}\right)$.

First of all, we give another proof of Corollary 2.5 using extremal partitions and Theorem 3.1. We use the inequality (3.1) to derive the inequality (2.25). Let $f$ be an arbitrary mapping with the properties asserted in the corollary. Without loss of generality, we again assume here that $\zeta_{1}=\overline{\zeta_{2}}$. We use the notations of Corollary 2.5. Let us fix the positive values of $t_{1}, t_{2}$. Let the pair of domains $\left(D_{1}^{*}, D_{2}^{*}\right)$ be an extremal pair for the minimum $\mathcal{M}\left(t_{1}, t_{2}, \zeta_{1}, \zeta_{2}\right)$ of the sum (3.1). Then the pair of domains $\left(f\left(D_{1}^{*}\right), f\left(D_{2}^{*}\right)\right)$ is an admissible pair for the minimum $\mathcal{M}\left(t_{1}, t_{2}, w_{1}, w_{2}\right)$. Therefore, the inequality

$$
t_{1}^{2} m\left(f\left(D_{1}^{*}\right), 0, w_{1}\right)+t_{2}^{2} m\left(f\left(D_{2}^{*}\right), 0, w_{2}\right) \geq t_{1}^{2} m\left(B_{1}^{*}, 0, w_{1}\right)+t_{2}^{2} m\left(B_{2}^{*}, 0, w_{2}\right),
$$

holds where $\left(B_{1}^{*}, B_{2}^{*}\right)$ is the extremal pair for the minimum $\mathcal{M}\left(t_{1}, t_{2}, w_{1}, w_{2}\right)$. By (1.3), (1.4) we deduce that

$$
\begin{aligned}
t_{1}^{2} m\left(f\left(D_{1}^{*}\right), 0,\right. & \left.w_{1}\right)+t_{2}^{2} m\left(f\left(D_{2}^{*}\right), 0, w_{2}\right) \\
= & t_{1}^{2} m\left(D_{1}^{*}, 0, w_{1}\right)+t_{2}^{2} m\left(D_{2}^{*}, 0, w_{2}\right) \\
& +\frac{t_{1}^{2}}{\pi} \log \left|f^{\prime}\left(\zeta_{1}\right)\right|+\frac{t_{2}^{2}}{\pi} \log \left|f^{\prime}\left(\zeta_{2}\right)\right|+\frac{\left(t_{1}+t_{2}\right)^{2}}{2 \pi} \log \alpha .
\end{aligned}
$$

Now we use Theorem 3.1 and calculate the moduli in (4.1) and (4.2). Thus, normalizing $t_{1}+t_{2}=1$, the inequalities (4.1) and (4.2) imply the inequality (2.25) in the assertion of Corollary 2.5.

Now we derive the extremal function $w=f^{*}(\zeta)$ and Equation (2.26). We examine the function $g_{1}(\zeta)$ given by (3.4) that maps the domain $D_{1}^{*}$ onto 
the upper half-plane $\mathbb{H}^{+}$, and the function $G_{1}$ that maps the domain $B_{1}^{*}$ onto $\mathbb{H}^{+}$. The function $G_{1}$ satisfies the differential equation

$$
t_{1}^{2}\left(\frac{G_{1}^{\prime}(w)}{G_{1}(w)}\right)^{2}=\pi^{2} \psi(w) .
$$

By analogy to (3.4), we obtain

$$
G_{1}(w)=\frac{\left(w-w_{1}\right)\left(w-w_{2}\right)^{t_{2} / t_{1}}}{w^{\frac{t_{1}+t_{2}}{2 t_{1}}}} w_{1}^{-t_{1} / 2} w_{2}^{-t_{2} / 2} e^{i\left(-\frac{\pi}{2} \frac{t_{1}+t_{2}}{t_{1}}\right)} .
$$

The superposition $G_{1}^{-1} \circ g_{1}(\zeta)$ after normalization $f^{*}(z)=\alpha z+\ldots$ gives us Equation (2.26) defined in the domain $D_{1}^{*}$. Now we repeat the same observations for $G_{2}(\zeta)$ that maps the domain $B_{2}^{*}$ onto $\mathbb{H}^{+}$and satisfies in $D_{2}^{*}$ the differential equation

$$
t_{2}^{2}\left(\frac{G_{2}^{\prime}(w)}{G_{2}(w)}\right)^{2}=\pi^{2} \psi(w) .
$$

The equation that we deduce for the superposition $G_{2}^{-1} \circ g_{2}(\zeta)$ is the same as for $f^{*}$. The condition $\alpha \in\left(0, \alpha_{0}\left(w_{1}, w_{2}\right)\right]$ follows from the subordination of domains. Therefore, the function $f^{*}$ is defined in the whole disk $\mathbb{D}$ and maps the extremal configuration $\left(D_{1}^{*}, D_{2}^{*}\right)$ onto the extremal configuration $\left(B_{1}^{*}, B_{2}^{*}\right)$. Thus, it gives the equality sign in (4.1-4.2), and, therefore, also in $(2.25)$.

Now we continue with bounded univalent functions $f: \mathbb{D} \rightarrow \mathbb{D}, f(0)=0$ that have the finite angular limits $f(\zeta) \in \mathbb{T}$ in the points of the arc of $\mathbb{T}$ connecting two points $\zeta_{1}, \zeta_{2} \in \mathbb{T}$ and the finite nonzero angular derivatives $f^{\prime}\left(\zeta_{1}\right), f^{\prime}\left(\zeta_{2}\right)$. This means that $f$ is continuous and analytic on this arc.

Lemma 4.1. Let $D\left(\theta_{1}, \theta_{2}\right)$ denote the triangle $\mathbb{D} \backslash\left(-e^{\frac{\theta_{1}+\theta_{2}}{2}}, 0\right]$ with a vertex at the origin and the opposite leg $\left\{z: z=e^{i \theta}, \theta \in\left[\theta_{1}, \theta_{2}\right]\right\}, 0<\theta_{2}-\theta_{1}<2 \pi$. Then, its reduced modulus with respect to the origin is

$$
m_{\Delta}\left(D\left(\theta_{1}, \theta_{2}\right), 0\right)=\frac{1}{2 \pi} \log \frac{2}{1-\cos \frac{\theta_{2}-\theta_{1}}{2}} .
$$

Proof. Obviously, one can rotate the configuration $z \rightarrow z e^{\frac{\theta_{2}+\theta_{1}}{2}}$ So, we assume $\theta_{1}=-\theta_{2}$. Then, we transfer the triangle $D\left(\theta_{1}, \theta_{2}\right)$ onto the triangle $D(-\pi, \pi)$ by the map $w(z)$ which is the solution of the equation

$$
\left(z+\frac{1}{z}-1-\cos \theta_{2}\right) \frac{2}{1-\cos \theta_{2}}=w+\frac{1}{w} .
$$

We derive that $\left|w^{\prime}(0)\right|=\frac{1-\cos \theta_{2}}{2}$ and, moreover, $m_{\Delta}(D(-\pi, \pi), 0)=0$. The formula of the change the reduced modulus of a triangle under conformal map and the mentioned rotation lead to the assertion of Lemma 4.2. 
The Löwner Lemma (see [10, Proposition 4.15]) yields that if $f(z)$ is a conformal map $f: \mathbb{D} \rightarrow \mathbb{D}, f(0)=0$ which has the finite angular limits $f\left(e^{i \theta}\right)=e^{i \sigma}, \theta \in\left[\theta_{1}, \theta_{2}\right], \sigma \in\left[\sigma_{1}, \sigma_{2}\right], 0<\theta_{2}-\theta_{1}<2 \pi, 0<\sigma_{2}-\sigma_{1}<2 \pi$, then

$$
\sigma_{2}-\sigma_{1} \geq \theta_{2}-\theta_{1}
$$

The equality sign is given by the identity mapping. Moreover, the following assertion is true.

Lemma 4.2. Let $f(z)$ be a conformal map $f: \mathbb{D} \rightarrow \mathbb{D}, f(0)=0$ which has the finite angular limits $f\left(e^{i \theta}\right)=e^{i \sigma}, \sigma \in\left[\sigma_{1}, \sigma_{2}\right], \theta \in\left[\theta_{1}, \theta_{2}\right], 0<\theta_{2}-\theta_{1}<$ $2 \pi, \sigma_{2}-\sigma_{1} \leq 2 \pi$. Then, the inequality

$$
\frac{1-\cos \frac{\theta_{2}-\theta_{1}}{2}}{1-\cos \frac{\sigma_{2}-\sigma_{1}}{2}} \leq\left|f^{\prime}(0)\right| \leq 1
$$

holds. The left-hand side inequality is sharp with the Pick extremal function $g_{\alpha}^{\varkappa}(z), \varkappa=\frac{\theta_{1}+\theta_{2}}{2}$ and $\sigma_{1}=\arg g_{\alpha}^{\varkappa}\left(e^{i \theta_{1}}\right), \sigma_{2}=\arg g_{\alpha}^{\varkappa}\left(e^{i \theta_{2}}\right)$. The right-hand side inequality is obvious due to the Schwarz lemma.

Proof. We use the results by A.Yu. Solynin [14] about the extremal partitions. Let us consider the triangle $f\left(D\left(\theta_{1}, \theta_{2}\right)\right)$ that is admissible in the family of all triangles in $\mathbb{D}$ with the vertex at the origin, with the inner angle $2 \pi$, and the opposite leg lying on $\mathbb{T}$ connecting the points $e^{i \sigma_{1}}, e^{i \sigma_{2}}$. The extremal triangle is $D\left(\sigma_{1}, \sigma_{2}\right)$. Then, due to the change of the reduced modulus under a conformal map $f(z)$ we have

$$
m_{\Delta}\left(D\left(\theta_{1}, \theta_{2}\right), 0\right)+\frac{1}{2 \pi} \log \left|f^{\prime}(0)\right| \geq m_{\Delta}\left(D\left(\sigma_{1}, \sigma_{2}\right), 0\right) .
$$

Lemma 4.1 leads to the inequality we need. The assertion about the sharpness is obvious.

Remark. The Pick function which gives the equality sign to (4.4) is subject to the choice of $\alpha \in\left(\sin ^{2} \frac{\theta_{2}-\theta_{1}}{4}, 1\right]$.

Suppose $0<\theta_{2}-\theta_{1}<\sigma_{2}-\sigma_{1}<\pi$. Set the functions $x(t), y(t)$ defined in the segment

$$
\tan \frac{\theta_{2}-\theta_{1}}{4} \leq t \leq \tan \frac{\sigma_{2}-\sigma_{1}}{4}
$$


by the formulas

$$
x(t)=\frac{2 t^{2}}{\left(1+t^{2}\right)\left(1-\cos \frac{\sigma_{2}-\sigma_{1}}{2}\right)} \exp \frac{2}{t}\left(\tan ^{-1} \frac{1}{t}-\frac{\pi}{2}+\frac{\theta_{2}-\theta_{1}}{4}\right),
$$

$$
y(t)=\frac{4}{\left(1+t^{2}\right)^{2}} \frac{\left(1-\cos \frac{\sigma_{2}-\sigma_{1}}{2}\right)^{2}}{\sin ^{2} \frac{\sigma_{2}-\sigma_{1}}{2} \sin ^{2} \frac{\theta_{2}-\theta_{1}}{2}} \exp 4 t\left(\frac{\pi}{2}-\frac{\theta_{2}-\theta_{1}}{4}-\tan ^{-1} \frac{1}{t}\right),
$$

and in the ray

$$
t \geq \tan \frac{\sigma_{2}-\sigma_{1}}{4}
$$

by the formulas

$$
\begin{aligned}
& x(t)=\exp \frac{1}{2 t}\left(\left(\theta_{2}-\theta_{1}\right)-\left(\sigma_{2}-\sigma_{1}\right)\right), \\
& y(t)=\frac{\sin ^{2} \frac{\sigma_{2}-\sigma_{1}}{2}}{\sin ^{2} \frac{\theta_{2}-\theta_{1}}{2}} \exp t\left(\left(\sigma_{2}-\sigma_{1}\right)-\left(\theta_{2}-\theta_{1}\right)\right) .
\end{aligned}
$$

The function $x(t)$ is continuous and increases in

$$
t \in\left[\tan \frac{\theta_{2}-\theta_{1}}{4}, \infty\right] \text { from } \frac{1-\cos \frac{\theta_{2}-\theta_{1}}{2}}{1-\cos \frac{\sigma_{2}-\sigma_{1}}{2}} \text { to } 1 .
$$

Theorem 4.3. Let $f(z)$ be a conformal map $f: \mathbb{D} \rightarrow \mathbb{D}, f(0)=0$ which has the finite angular limits $f\left(e^{i \theta}\right)=e^{i \sigma}, \theta \in\left[\theta_{1}, \theta_{2}\right], \sigma \in\left[\sigma_{1}, \sigma_{2}\right]$ and the finite nonzero angular derivatives $f^{\prime}\left(\zeta_{1}\right), f^{\prime}\left(\zeta_{2}\right)$ in two points $\zeta_{1}=e^{i \theta_{1}}, \zeta_{2}=e^{i \theta_{2}}$, $0<\theta_{2}-\theta_{1}<\pi$. We suppose that $\theta_{2}-\theta_{1}<\sigma_{2}-\sigma_{1} \leq \pi$, fixed and we consider all such functions. If $\alpha:=\left|f^{\prime}(0)\right|$, then there exists a unique real number

$$
t^{*} \in\left[\tan \frac{\theta_{2}-\theta_{1}}{4}, \infty\right]
$$

such that $x\left(t^{*}\right)=\alpha$ and $\left|f^{\prime}\left(\zeta_{1}\right) f^{\prime}\left(\zeta_{2}\right)\right| \geq y\left(t^{*}\right)$. This means that the range of the system of functionals $\left(\left|f^{\prime}(0)\right|,\left|f^{\prime}\left(\zeta_{1}\right) f^{\prime}\left(\zeta_{2}\right)\right|\right)$ as the set in the $\mathbb{R}^{2}$-plane is bounded from the below by an arc of the curve $\Gamma^{-}$which is given parametrically by $(x(t), y(t))$ with $t \in\left[\tan \frac{\theta_{2}-\theta_{1}}{4}, \infty\right]$.

Proof. In order to prove this theorem we use the inequality (3.6) and the extremal partition of $\mathbb{D}$ by digons and circular domains. First we note that the conditions of Theorem 4.3 satisfy the inequality (4.3). This implies that the intervals (4.5), (4.8) are well-defined for $\sigma_{1}, \sigma_{2}$ given by the conditions of Theorem 4.3. The conditions of Theorem 4.3 imply that $f: \mathbb{D} \rightarrow \mathbb{D}, f(0)=$ 0 , and the function $f$ has the finite angular limits $f\left(\zeta_{1}\right)=e^{i \sigma_{1}}, f\left(\zeta_{2}\right)=e^{i \sigma_{2}}$ with a fixed value of $\sigma_{2}-\sigma_{1}$. We consider all such functions with a fixed $\left|f^{\prime}(0)\right|=\alpha$. The function $x(t)$ increases. Moreover, Lemma 4.2 yields that 
there exists a unique $t=t^{*}$ such that $x\left(t^{*}\right)=\alpha$. Let $D_{1}^{*}$ and $D_{2}^{*}$ be extremal circular and strip domains respectively in the problem of $\mathcal{M}_{2}^{(1)}\left(t^{*}, 1,0, \zeta_{1}, \zeta_{2}\right)$. Then, the pair of the domains $f\left(D_{1}^{*}\right), f\left(D_{2}^{*}\right)$ have the reduced moduli

$$
m\left(f\left(D_{1}^{*}\right), 0\right)=m\left(D_{1}^{*}, 0\right)+\frac{1}{2 \pi} \log \alpha,
$$

and

$$
m\left(f\left(D_{2}^{*}\right), e^{i \sigma_{1}}, e^{i \sigma_{2}}\right)=m\left(D_{2}^{*}, \zeta_{1}, \zeta_{2}\right)+\frac{1}{\pi} \log \left|f^{\prime}\left(\zeta_{1}\right) f^{\prime}\left(\zeta_{2}\right)\right| .
$$

This pair $f\left(D_{1}^{*}\right), f\left(D_{2}^{*}\right)$ is admissible in the problem of $\mathcal{M}_{2}^{(m)}\left(t^{*}, 1,0, f\left(\zeta_{1}\right)\right.$, $\left.f\left(\zeta_{2}\right)\right)$ for $m=1$ or 2 . In our case $\sigma_{2}-\sigma_{1}<\pi$, therefore, $m=1$. This leads to the inequality

$$
\begin{aligned}
& \left(t^{*}\right)^{2} m\left(f\left(D_{1}^{*}\right), 0\right)-m\left(f\left(D_{2}^{*}\right), e^{i \sigma_{1}}, e^{i \sigma_{2}}\right) \\
& \quad \leq\left(t^{*}\right)^{2} m\left(B_{1}^{*}, 0\right)-m\left(B_{2}^{*}, e^{i \sigma_{1}}, e^{i \sigma_{2}}\right)=\mathcal{M}_{2}^{(1)}\left(t^{*}, 1,0, f\left(\zeta_{1}\right), f\left(\zeta_{2}\right)\right)
\end{aligned}
$$

where $B_{1}^{*}$ and $B_{2}^{*}$ are the pair of extremal domains for $\mathcal{M}_{2}^{(1)}\left(t^{*}, 1,0, e^{i \sigma_{1}}, e^{i \sigma_{2}}\right)$. Given $t$ from the interval (4.5) we calculate the reduced moduli with $x(t)$, $y(t)$ provided by the formulas (4.6-4.7) and deduce that $m\left(B_{1}^{*}, 0\right)=m\left(D_{1}^{*}, 0\right)$ $+\frac{1}{2 \pi} \log x\left(t^{*}\right)$ and $m\left(B_{2}^{*}, e^{i \sigma_{1}}, e^{i \sigma_{2}}\right)=m\left(D_{2}^{*}, \zeta_{1}, \zeta_{2}\right)+\frac{1}{\pi} \log y\left(t^{*}\right)$. Taking into account $x\left(t^{*}\right)=\alpha$ we finally derive from (4.11-4.13) the inequality $\left|f^{\prime}\left(\zeta_{1}\right) f^{\prime}\left(\zeta_{2}\right)\right| \geq y\left(t^{*}\right)$ which proves the assertion of Theorem 4.3 about the estimate and the lower boundary curve $\Gamma^{-}$.

\section{Remarks.}

1. One can deduce similar results for other distributions of $\theta_{1}, \theta_{2}, \sigma_{1}, \sigma_{2}$ using $\mathcal{M}_{2}^{(2)}\left(t, 1,0, \zeta_{1}, \zeta_{2}\right)$.

2. From Theorem 2.1, formula (2.4) we get the inequality

$$
2 \log \frac{1}{\alpha} \log \left|\frac{f^{\prime}\left(\zeta_{1}\right) f^{\prime}\left(\zeta_{2}\right)\left(f\left(\zeta_{1}\right)-f\left(\zeta_{2}\right)\right)^{2}}{\left(\zeta_{1}-\zeta_{2}\right)^{2}}\right| \geq\left(\arg \frac{f\left(\zeta_{1}\right)}{\zeta_{1}}-\arg \frac{f\left(\zeta_{2}\right)}{\zeta_{2}}\right)^{2} .
$$

Theorem 4.3 implies that the inequality (4.14) describes a part of the curve $\Gamma^{-}$which is parameterized by $t \in\left[\tan \frac{\sigma_{2}-\sigma_{1}}{4}, \infty\right]$. The rest of $\Gamma^{-}$lies above the curve given by (4.14) because the extremal function does not give the equality sign in (4.14), say the extremal function maps $\mathbb{D}$ onto $\mathbb{D}$ minus a fork-shaped slit which can not be represented by Theorem 2.3.

3. One could assume $f\left(\zeta_{1}\right)=\zeta_{1}, f\left(\zeta_{2}\right)=\zeta_{2}$ which makes no use Theorem 4.3 instead of (4.14) but this assumption leads only to the trivial case of the identical map. Thus, the part of $\Gamma^{-}$which is not given by (4.14) is always valuable.

Corollary 4.4. Let $f(z)$ be a conformal map $f: \mathbb{D} \rightarrow \mathbb{D}, f(0)=0$ which has the finite angular limits $f\left(e^{i \theta}\right)=e^{i \sigma}, \theta \in\left[\theta_{1}, \theta_{2}\right], \sigma \in\left[\sigma_{1}, \sigma_{2}\right], 0<$ 
$\sigma_{2}-\sigma_{1}<2 \pi$ and the finite nonzero angular derivatives $f^{\prime}\left(\zeta_{1}\right), f^{\prime}\left(\zeta_{2}\right)$ in two points $\zeta_{1}=e^{i \theta_{1}}, \zeta_{2}=e^{i \theta_{2}},\left|\theta_{2}-\theta_{1}\right| \leq \pi$. Then the inequality

$$
\left|f^{\prime}\left(\zeta_{1}\right) f^{\prime}\left(\zeta_{2}\right)\right| \geq \tan ^{2} \frac{\sigma_{2}-\sigma_{1}}{4} \cot ^{2} \frac{\theta_{2}-\theta_{1}}{4}
$$

holds with the equality sign for the identical map as well as for the canonical $\operatorname{map} g_{\alpha}^{\varkappa}(z), \varkappa=\frac{\theta_{1}+\theta_{2}}{2}$ with $\alpha=\sin ^{2} \frac{\theta_{2}-\theta_{1}}{4} \sin ^{-2} \frac{\sigma_{2}-\sigma_{1}}{4}$.

Proof. Let $f$ be a map satisfying the conditions of Corollary 4.4. Then, by analogy to (4.11-4.13), we deduce that the inequality

$$
\begin{aligned}
& \mathcal{M}_{2}^{(1)}\left(t, 1,0, e^{i \theta_{1}}, e^{i \theta_{2}}\right)+\frac{t^{2}}{2 \pi} \log \left|f^{\prime}(0)\right|-\frac{1}{\pi} \log \left|f^{\prime}\left(\zeta_{1}\right) f^{\prime}\left(\zeta_{2}\right)\right| \\
& \leq \mathcal{M}_{2}^{(1)}\left(t, 1,0, e^{i \sigma_{1}}, e^{i \sigma_{2}}\right)
\end{aligned}
$$

holds for all $t>0$. Considering $t \rightarrow 0$ we deduce the inequality (4.15).

\section{References}

[1] V.N. Dubinin, Symmetrization in the geometric theory of functions of a complex variable, Uspekhi Mat. Nauk, 49(1) (1994), 3-76; English transl. in Russian Math. Surveys, 49(1) (1994), 1-79, MR 96b:30054, Zbl 0830.30014.

[2] E.G. Emel'yanov, On problems on extremal decomposition, Zap. Nauchn. Sem. Leningrad. Otdel. Mat. Inst. Steklov (LOMI), 154 (1986), 76-89; English transl., J. Soviet Math., 43(4) (1988), 2558-2566, Zbl 0608.30026.

[3] G.M. Goluzin, Geometric Theory of Functions of a Complex Variable, 2nd ed., Nauka, Moscow, 1966; English transl., Transl. Math. Monogr., 26, Amer. Math. Soc., Providence, RI, 1969, MR 40 \#308, Zbl 0183.07502.

[4] J. Jenkins, On the existence of certain general extremal metrics, Ann. of Math., 66(3) (1957), 440-453, MR 19,845g, Zbl 0082.06301.

[5] _ Univalent Functions and Conformal Mapping, Second corrected edition, Springer-Verlag, 1965, MR 20 \#3288, Zbl 0106.04801.

[6] G.V. Kuz'mina, Moduli of families of curves and quadratic differentials, Trudy Mat. Inst. Steklov., 139 (1980), 1-240; English transl., Proc. Steklov Inst. Math., 1 (1982), VII+231 pp., MR 84j:30038b, Zbl 0491.30013.

[7] _ On extremal properties of quadratic differentials with strip-shaped domains in the structure of the trajectories, Zap. Nauchn. Sem. Leningrad. Otdel. Mat. Inst. Steklov. (LOMI), 154 (1986), 110-129; English transl., J. Soviet Math., 43(4) (1988), 2579-2591, MR 88h:30033, Zbl 0664.30020.

[8] J.E. McMillan, Boundary behavior of a conformal mapping, Acta Math., 123 (1969), 43-67, MR 41 \#1981, Zbl 0222.30006.

[9] Ch. Pommerenke, Univalent Functions, with a chapter on quadratic differentials by G. Jensen, Vandenhoeck \& Ruprecht, Göttingen, 1975, MR 58 \#22526, Zbl 0298.30014.

[10] , Boundary Behaviour of Conformal Maps, Springer, Berlin, 1992, MR 95b:30008, Zbl 0762.30001. 
[11] Ch. Pommerenke and A. Vasil'ev, On bounded univalent functions and the angular derivative, Ann. Univ. Maria Curie-Slodowska. Ser. A., 8 (2000), 79-106, MR 2002d:30019.

[12] M. Schiffer and O. Tammi, On the coefficient problem for bounded univalent functions, Trans. Amer. Math. Soc., 140 (1969), 461-474, MR 39 \#7088, Zbl 0183.07701.

[13] A.Yu. Solynin, Dependence of the modulus problem for a family of several classes of curves on parameters, Zap. Nauchn. Sem. Leningrad. Otdel. Mat. Inst. Steklov. (LOMI), 144 (1985), 136-145; English transl., J. Soviet Math. 38(4) (1987), 21312139 .

[14] _ Modules and extremal metric problems, Algebra i Analiz, 11(1) (1999), 3-86; English transl. St. Petersburg Math. J., 11(1) (2000), 1-70, Zbl 0935.30019.

[15] K. Strebel, Quadratic Differentials, Springer-Verlag, 1984, MR 86a:30072, Zbl 0547.30001.

[16] A. Vasil'ev, Moduli of Families of Curves for Conformal and Quasiconformal Mappings, Lect. Notes in Math., 1788, Springer-Verlag, Berlin-New York, 2002.

[17] _ On the distortion under bounded univalent functions with the angular derivative fixed, Complex Var. Theory Appl., 47(2) (2002), 131-147, CMP 1892514.

Received December 6, 2000 and revised January 13, 2002. The second author was supported by FONDECYT (Chile), Grants \#1010093 and \#1020067.

FACHBEReich Mathematik

TeChnische Universität Berlin

D-10623 Berlin, Germany

E-mail address: pommeren@math.tu-berlin.de

Departamento de Matemática

Universidad TÉcnica Federico Santa María

Casilla 110-V, Valparaíso, Chile

E-mail address: avassili@mat.utfsm.cl 